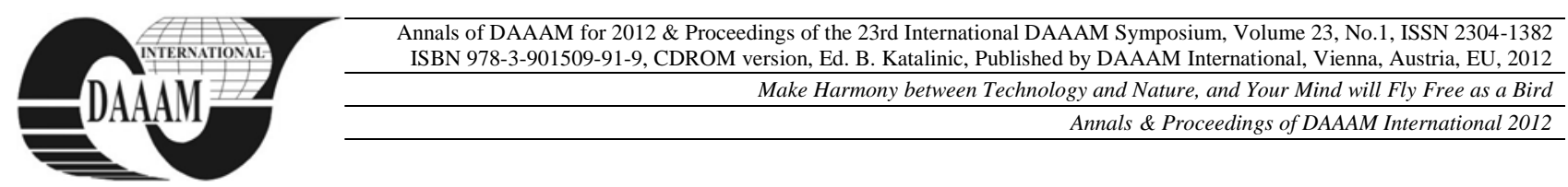

\title{
GARMENT SEAM STRENGTH DEPENDING ON NEEDLE SIZE AND STITCH LENGTH
}

\author{
FIRST ROGALE, S[njezana]; BOBOVCAN MARCELIC, M[artina]; ROGALE, D[ubravko]; \\ DRAGCEVIC, Z[vonko] \& NIKOLIC, G[ojko]
}

\begin{abstract}
Joining patterns into an article of clothing are usually performed using sewing machines where sewing threads are drawn between different material layers making different machine stitches with necessary properties. Appropriate choice of the type of sewn seam, machine sewing stitch, needle and sewing thread allows making joints with accurately specific elasticity and strength. This kind of joining is relatively inexpensive and reliable. Most of the garment seams are subject to forces. If seam strength is small, the seam may break. Each joint should have minimum strength. This paper explores optimal parameters of sewing needle size and the number of seam stitches for three material types in order that a qualitative and strong seam is obtained.
\end{abstract}

Keywords: sewing needle size, number of stitches, seam strength

\section{INTRODUCTION}

Besides the looper, the sewing machine needle is an element that directly participates in creating sewing stitches. Its task is to penetrate the sewing material with its point, to pass through the material into the bottom position and to pull the sewing thread that is required for forming stitches. Afterwards, the needle leaves the material into the upper position and due to thread friction with the material a loop is formed. The loop is taken by the looper, and the formation of a sewing machine stitch starts [1].

Sewing machine needle consists of several basic parts. The shank is inserted into the needle bar; its form can be round, flat or with flat notches. The shaft of a sewing machine needle is the area from the bottom of the shank to the point. The groove is in the side of the needle leading to the eye. A long and a short groove and a scarf are in the needle. The long groove is a place for the thread to lay into the needle and protects the thread when it penetrates the sewing material. The cross-section of the long groove is about $40 \%$ of the cross-section of the sewing needle. The short groove takes the needle thread after threading the thread through the eye and protects it against too high a friction while the needle eye is within the sewing material. The scarf is above the needle eye and allows the bobbin case hook to intersect with the upper thread and form stitches. It also allows the safe passage of the tip of the looper along the needle [2]. The eye of the needle carries the needle thread so the machine can keep forming stitches. Due to the speed of the thread through the eye, it is necessary that it is precisely made, because, depending on stitch length, sewing stitch type and sewing machine type, the thread passes through the needle eye 40 to 60 times before it is sewn into the seam.
The point of the needle is the first contact with the fabric and responsible for how the needle pierces the fabric.

According to standard DIN 5325 sewing machine needles are designated based on sewing needle thickness using the metric system, with the abbreviation $\mathrm{Nm}$. The metric number $\mathrm{Nm}$ corresponds to the hundredfold diameter of the sewing needle shaft "d" in mm. Sewing needle thickness is a very important component since sewing material thickness, thread thickness and needle thickness are three decisive factors for the quality of the seam.

The hardness of the needle surface should be as great as possible when modern sewing machine needles are used for sewing technical textiles. High values of the hardness of sewing machine needles are necessary to increase the resistance to needle wear and to deformations of the needle point caused by short contacts with loopers of the sewing machine, throat plate or with the parts of the sewing machine feed system. It was found that the hardness of a material is correlated with some other mechanical properties, which are primarily related to compressive strength, resistance to abrasion and other mechanical properties. It is customary that the surface hardness of sewing needles is measured by the Vickers hardness test method, and the measurement units are marked with HV. A square base pyramid shaped diamond is used for testing in the Vickers scale. Hardness testing slightly damages the surface of the item to be tested, and such testing might be called nondestructive testing. Another advantage is that the test does not need a separate test specimen. Hardness testing is performed on the surface of the sewing machine needle.

According to Groz - Beckert [1], machine sewing needles made of hard steel have hardness lower than $1000 \mathrm{HV}$, and those with chrome finish about $1100 \mathrm{HV}$. Needles coated with Wolfram (tungsten) carbide having surface hardness lower than $2000 \mathrm{HV}$, while the highest hardness of sewing machine needles is achieved using titanium nitride. The hardness of titanium nitride coated surface is about $2500 \mathrm{HV}$ and it is up to the highest value of surface hardness of sewing machine needles [3].

Surface coating of machine sewing needles with different metal compounds is carried out using Chemical Vapor Deposition (CVD) or Physical Vapor Deposition (PVD). Chemical vapor deposition is a process carried out at high temperatures in chemical equilibrium. Physical Vapor Deposition takes place far 
out of chemical equilibrium and at relatively low temperatures (about $500{ }^{\circ} \mathrm{C}$ ). Product surfaces are coated with metal compounds containing some of metalloids $(\mathrm{C}$, $\mathrm{N}, \mathrm{O}, \mathrm{B}, \mathrm{P}, \mathrm{S})$. These compounds have a simple grid structure, and are characterized by high hardness, wear resistance, low friction coefficient, good thermal conductivity and resistance to chemical attack. The hardness of such coated surfaces range from 3,800 to $5,000 \mathrm{HV}$.

The surface clad with platinum with titanium nitride using the PVD process has an optimum film thickness from 2 to 4 microns, and a relatively low friction coefficient ( $\mathrm{TiN} /$ steel is 0.4 to 0.7 , and $\mathrm{TiN} / \mathrm{TiN}$ is from 0.13 to 0.2 ). Temperature resistance amounts up to $550{ }^{\circ} \mathrm{C}$, with high resistance to acids and alkalis. The surface coating of titanium nitride has no toxicity, and is characterized by a golden yellow color of the surface coating [2].

Needles whose surface is coated with titanium nitride coating have improved sewing properties reflected in a reduced number of broken needles, greater security regarding skipped stitches, fewer thread breaks, a higher level of protection of the work piece and the looper tip, which is reflected in increased productivity and reduced production costs.

For sewing machine needles phosphorous treatment can be applied to their surface and after treatment with Teflon coating. Such a is used by the company Schmetz [3] for Blukold needles. This type of needle is used for sewing textile fabrics made from synthetic polymers or blends with a higher content of such fibers. They are also used for sewing materials with special finishing characterized by the fact that they are deposited on the sewing machine needle and cause heating of needles due to increased friction and difficult thread movement. In these needles the adhesive bonding of fine particles with a low softening point or melting is prevented. Surface treatment of the machine sewing needle with phosphor and Teflon prevents adhesive bonding of thread or yarn residues or finishing agents on the surface of the needle. The surface of such a needle remains clean much longer, so skipped stitches or thread breaks are much less common than in for example chrome needles [4].

For other conventional needles mechanical and chemical polishing of needle surfaces is performed to reduce friction coefficient, penetration force and heating up. This especially applies to the eye of the needle where thread loads are the highest. This roughness can significantly damage the sewing thread causing tiny knots and snarls on the thread, resulting in thread breakage. Polishing the needle eye using a polishing thread a significantly better smoothness of the needle eye is achieved, which is reflected in a significantly lower number of damages and thread breakages during sewing.

It can be assumed that the further development of surface treatment techniques reflect on the development of machine sewing needles to enhance the properties of all the elements in the sewing system: thread, needle, work piece and sewing machine.

The development of the needle point and different applications with respect to the type of the needle point are important for sewing machine needles.

Needles used for sewing textile materials generally have round conical points and round ball points. Needles for sewing leather, laminates and films have blades at the needle point, which lie under a certain angle in relation to the sewing direction.

Sewing machine conical point needles for sewing textile materials can have:

- very sharp conical point,

- medium sharp conical point,

- slightly sharp conical point.

Swing machine ball point needles can have:

- small ball point,

- medium ball point,

- large ball point,

- special ball point.

Figure 1 shows shapes of needle points with their letter marks [5].

Very sharp conical point (RS, SPI) is mostly used for sewing machine needles which are used on special blind stitch sewing machines. Their sharp plays a noticeably great role in prick stitching tangential penetration into the material, whereby weft and warp threads should not be displaced significantly. These point shapes have a similar function in tacking parts where the aesthetic component is very visible or a strictly specified seam path is required. For this point type it is characteristic that they to a very small extent displace warp and weft threads. Thus, a sewing machine stitch is formed at the point of needle penetration, resulting in a flat seam with great accuracy and aesthetic component [5].

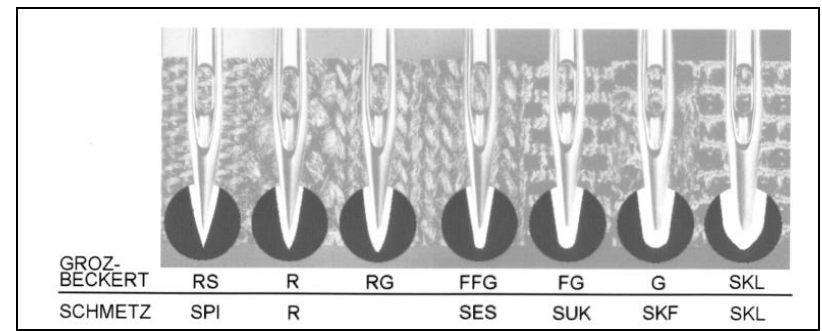

Fig. 1. Shapes of sewing machine needle points

Medium sharp conical needle point ( $\mathrm{R}$ ) is mostly used in technological operations of sewing using lock stitch sewing machines (type 301), and it is most suitable for sewing woven fabrics.

Slightly sharp conical needle point (RG) is recommendable for all types of chain stitches (type 401), especially for knitted fabrics, and for sewing on automatic machines. The point of the conventional needle may damage an incorrectly positioned button or it may come to bending or breaking the sewing machine needle. An additional advantage of this needle point type is its possible application in sewing labels and bar tacking [3, 4]. 
Small ball point (FFG, SES) is most suitable for sewing all types of knitted fabrics and synthetic materials using lock stitch, but it can also be used in case of chain stitches.

Medium ball point (FG, SUK) is recommendable for highly elastic textile materials with rubber and/or elastomeric components.

Large ball point (G, SKF) is used in sewing bulkier and coarser knitted fabrics and open-structured knitted fabrics with elastic properties.

Special ball point (SKL) is actually a combination of obtuse cone and ball point for sewing bulky knitted fabrics and elastomeric woven fabrics (so-called power stretch) with a great portion of elastomeric components.

Sewing stitch is the basic unit of interlacing the thread formed from one or more threads or thread loops, which by themselves or together are interlaced or intertwined.. Sewing stitch can be formed without a material, within the material, through the material and in the sewing material. A series of sewing stitches or many types of sewing stitches on one or more layers of the sewing material make a sewn seam $[6,7]$.

\section{RESULTS}

Three different material samples were used to test seam strength: material no. $1(100 \%$ cotton in twill weave $0,309 \mathrm{~mm}$ thick), material no. 2 (100\% viscose fiber $0,181 \mathrm{~mm}$ thick) and material no. 3 (lining $100 \%$ PES $102 \mathrm{~mm}$ thick). The thread Astra made by Coats denoted 080 09700, three Schmetz needle sizes, $70 \mathrm{Nm}$, $90 \mathrm{Nm}$ and $110 \mathrm{Nm}$ and three different material feeds 2 $\mathrm{mm}, 2.5 \mathrm{~mm}$ and $3 \mathrm{~mm}$ were used in testing. The type of the needle point in all needles was point type SPI. Sewing speed was adjusted in the process microcomputer at 1000 stitches/min.

For each material 10 samples were made which were used in testing breaking strength on the tensile tester Sauter TVM. The extension speed of the tensile tester was $70 \mathrm{~mm} / \mathrm{min}$.

Breaking length of the test specimen $1_{\max }$ on maximum force $F_{\max }$ which could be measured during testing, and which is seam breaking force, was determined from the results obtained. From the results obtained, the mean maximum force for all samples regarding needle size and number of stitches. The results are shown in Tab.1.

Figure 2 shows 3D dependence of average maximum breaking force $\left(\mathrm{F}_{\max } / \mathrm{N}\right)$ causing the seam to break on needle size $(\mathrm{Nm})$ and stitch length for the sample made of $100 \%$ cotton in twill weave.

Figure 4 shows 3D dependence of average maximum breaking force $\left(\mathrm{F}_{\max } / \mathrm{N}\right)$ causing the seam to break on needle size $(\mathrm{Nm})$ and stitch length for the sample made of lining $100 \%$ PES.

\begin{tabular}{|r|r|r|r|r|}
\hline \multirow{4}{*}{ Nm } & $\begin{array}{r}\text { Stitch } \\
\text { length }\end{array}$ & \multicolumn{1}{c|}{2} & \multicolumn{1}{c|}{2,5} & \multicolumn{1}{c|}{3} \\
\cline { 2 - 5 } & $\begin{array}{l}\text { The } \\
\text { samples }\end{array}$ & & & \\
\hline \multirow{3}{*}{70} & S1 & 185,30 & 133,70 & 107,00 \\
\cline { 2 - 5 } & S2 & 96,10 & 94,10 & 91,60 \\
\cline { 2 - 5 } & S3 & 206,00 & 203,17 & 147,36 \\
\hline \multirow{3}{*}{90} & S1 & 186,00 & 149,00 & 117,70 \\
\cline { 2 - 5 } & S2 & 97,63 & 95,78 & 93,50 \\
\cline { 2 - 5 } & S3 & 217,11 & 195,22 & 146,33 \\
\hline \multirow{3}{*}{110} & S1 & 133,60 & 126,60 & 109,00 \\
\cline { 2 - 5 } & S2 & 80,8 & 89,75 & 94,00 \\
\cline { 2 - 5 } & S3 & 238,25 & 205,50 & 156,00 \\
\hline
\end{tabular}

Tab. 1. Values of average maximum forces with regard to needle size and number of stitches

Figure 3 shows 3D dependence of average maximum breaking force causing the seam to break on needle size $(\mathrm{Nm})$ and stitch length for the sample made of $100 \%$ viscose.

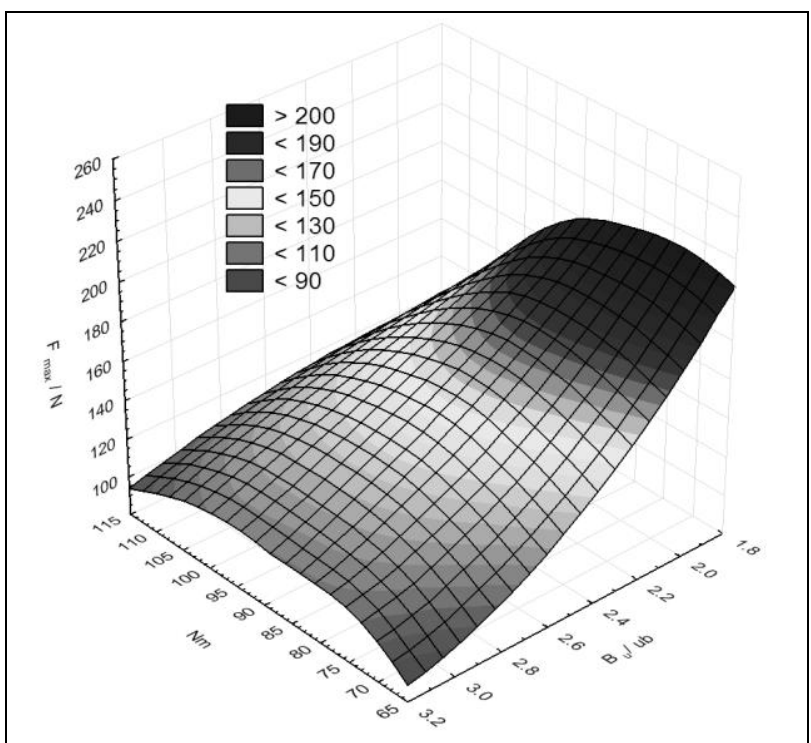

Fig. 2. 3D dependence of average maximum breaking force $\left(\mathrm{F}_{\max } / \mathrm{N}\right)$ causing the seam to break on needle size $(\mathrm{Nm})$ and stitch length for the sample made of $100 \%$ cotton in twill weave

The 3D diagrams show that average maximum breaking strengths for the samples made of $100 \%$ cotton in twill weave and the samples made of $100 \%$ viscose $\left(\mathrm{F}_{\max } / \mathrm{N}\right)$ increase with increasing needle size to $90 \mathrm{Nm}$, and afterwards they decline, and that they increase with reducing stitch length. For the samples made of lining $100 \%$ PES maximum forces $\left(\mathrm{F}_{\max } / \mathrm{N}\right)$ with increasing needle size and with reducing stitch length.

The obtained results show that for the samples made of $100 \%$ cotton in twill weave the optimal needle size is $90 \mathrm{Nm}$ and the material feed $2 \mathrm{~mm}$, because the maximum force in the amount of $186,0 \mathrm{~N}$ is required to prevent damage or breakage of the seam. The weakest seam is obtained using a needle size of $70 \mathrm{Nm}$ and the material feed of $3 \mathrm{~mm}$, where the force that causes a breakage or damage of the seam is $107 \mathrm{~N}$.

For the samples made of $100 \%$ viscose fiber 0,181 $\mathrm{mm}$ thick the optimal needle size is also $90 \mathrm{Nm}$ for the material feed of $2 \mathrm{~mm}$ because the highest force is necessary in order to damage or to break the seam 
amounting to $97,63 \mathrm{~N}$. The weakest seam is obtained when using needle size $110 \mathrm{Nm}$ and the material feed of $2 \mathrm{~mm}$ whereby the force causing breakage or damage of the seam amounts to $80,8 \mathrm{~N}$.

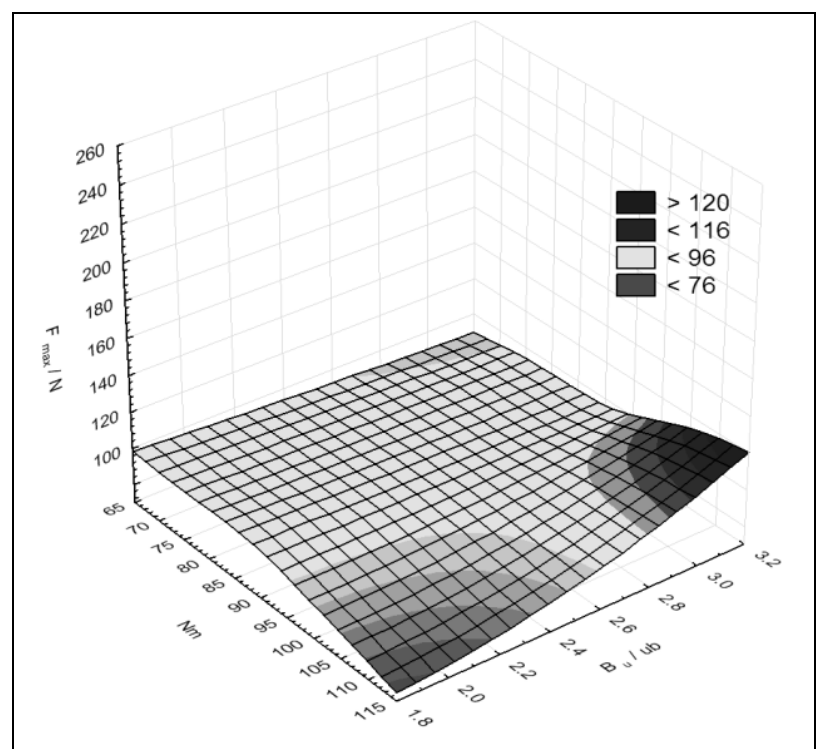

Fig. 3. 3D dependence of average maximum breaking force causing the seam to break on needle size $(\mathrm{Nm})$ and stitch length for the sample made of $100 \%$ viscose

For the samples made of lining 100\% PES $102 \mathrm{~mm}$ thick the optimal needle size is $110 \mathrm{Nm}$ and the material feed is $2 \mathrm{~mm}$ because the highest force to cause damage or breakage of the seam amounts to $238,25 \mathrm{~N}$. The weakest seam is obtained using needle size $90 \mathrm{Nm}$ and the material feed of $3 \mathrm{~mm}$ whereby the force causing breakage or damage of the seam is $146,33 \mathrm{~N}$.

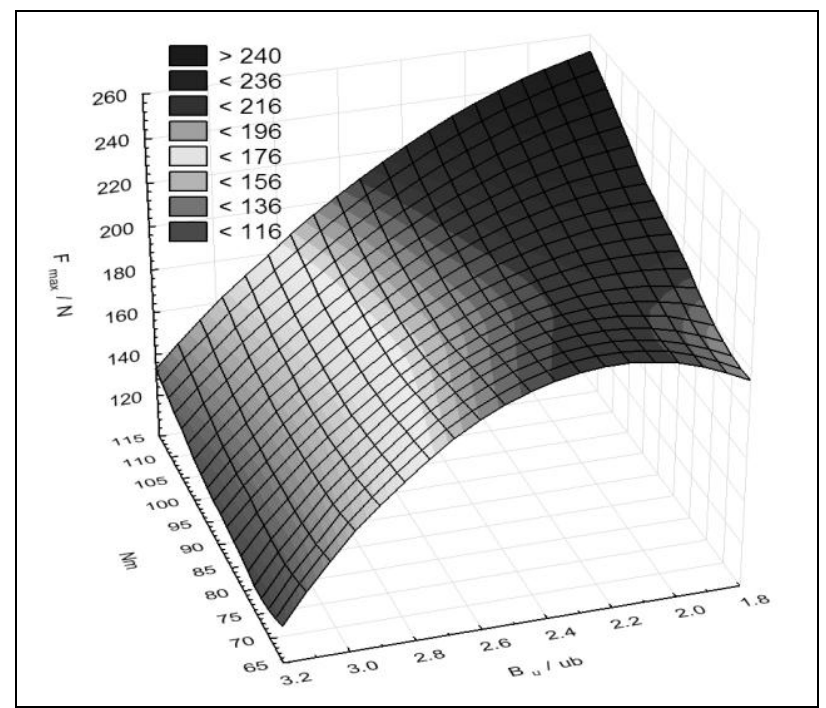

Fig. 4. 3D dependence of average maximum breaking force $\left(\mathrm{F}_{\max } / \mathrm{N}\right)$ causing the seam to break on needle size $(\mathrm{Nm})$ and stitch length for the sample made of lining $100 \%$ PES

From the research carried out it is observable that in the technological process of sewing care should be taken of choosing sewing thread fineness, needle size and number of stitches in the seam with regard to the sewing material to reduce the number of seam damages. These damages can be caused by machine sewing needle or penetration force of the sewing needle (holes, loop damages, damaged warp or weft, melting thread due to the needle temperature, etc...). Poor seam strength can be the consequence of the mismatch of various technological parameters, overload or incorrect handling, damages caused by thread breakage, because of incompatibility with sewing needle size, inadequate choice of sewing thread in relation to the applied type of sewing stitch and sewing needle size, too great sewing speed, the tension of the lower and upper thread, heating, etc. Stitch skipping should be avoided due to inadequate technological parameters, seam breakage or sewing with an unsuitable stitch type, tension of the upper and lower thread, too high or too low horizontal or vertical pressure of the presser foot. The above statements prove that the quality of the seam and its strength can be influenced by many factors, of which the technologists must take care during the sewing process.

\section{CONCLUSION}

The quality of the garment is reflected among other things in seam quality, its strength and appearance. Due to wearing and washing the seam breaks due to the weakening of material and / or thread. It also comes to stretching and then breaking the seam due to wearing because the seam is subjected to the force (e.g. when wearing tight clothes, during the movement of the body into a sitting position, etc.). However, seam strength and quality depends on needle size and number of stitches that are determined in the sewing process.

This is the reason why optimal parameters of sewing needle size and number of stitches in the seam for three types of materials in order to obtain a qualitative and strong seam were the topic of this research paper.

\section{REFERENCES}

[1] Žunić-Lojen, D.; Geršak. J. (2001). Study of the tensile force of thread in relation to its pre-tension. International Journal of Clothing Science and Technology, Vol. 13, No. 3/4, (12/2001) pp. 240-250, ISSN 0955-6222

[2] Firšt Rogale, S.; Dragčević, Z.; Rogale, D. (2003). Determining reaction abilities of sewing machine operators in joining curved seams. International Journal of Clothing Science and Technology, Vol. 15, No. 3/4, (12/2003)pp.179-188, ISSN 0955-6222

[3] http://www.groz-beckert.com (2001). Special application needle SAN 5 Developed for the sewing of technical textiles, GrozBeckert INFO, SMN 10, Germany, Accessed on: 2012-06-07

[4] http://www.schmetz.com (2001). Needles for avoiding specific sewing problems: Special needle "SERV 7" \& Blukold needle, Schmetz, Herzogenrath, Germany, Accessed on: 2012-06-07

[5] Rogale, D.; Dragčević, D. (2002). Making-up Technique of Technical Textile, Tekstil, Vol. 51, No. 2; (02/2002), pp.64-77, ISSN $0492-5882$

[6] Webster, J. (2009). Stitches and Seams, Textile Institute publication, ISBN 1870812735, Manchester

[7] Firšt Rogale, S., Dragčević, Z. (2001). Mathematical modeling of the system for defining hand-machine sewing sub-operation times, Proceedings of the 12th International DAAAM Symposium, Katalinic, B. (Ed.), pp. 143-144, ISBN 3-901509-19-4, Jena, Germany, 24-27 th October 2001 